\title{
The Smart Nonconserver: Preschoolers Detect Their Number Conservation Errors
}

\author{
Wim De Neys, ${ }^{1,2,3}$ Amélie Lubin, ${ }^{1,2,3}$ and Olivier Houdé ${ }^{1,2,3}$ \\ ${ }^{1}$ CNRS, Unité 8240, LaPsyDÉ, 75005 Paris, France \\ ${ }^{2}$ Université Paris Descartes, Sorbonne Paris Cité, Unité 8240 LaPsyDÉ, Laboratoire Alfred Binet 46, Rue Saint Jacques, \\ 75005 Paris, France \\ ${ }^{3}$ Université de Caen Basse-Normandie, Normandie Université, Unité 8240 LaPsyDÉ, Esplanade de la Paix, 14032 Caen, France
}

Correspondence should be addressed to Wim De Neys; wim.de-neys@parisdescartes.fr

Received 16 August 2013; Revised 22 November 2013; Accepted 21 December 2013; Published 12 February 2014

Academic Editor: Andrew N. Meltzoff

Copyright (c) 2014 Wim De Neys et al. This is an open access article distributed under the Creative Commons Attribution License, which permits unrestricted use, distribution, and reproduction in any medium, provided the original work is properly cited.

\begin{abstract}
Classic developmental studies have established that children's number conservation is often biased by misleading intuitions. However, the precise nature of these conservation errors is not clear. A key question is whether children detect that their erroneous conservation judgment is unwarranted. The present study focuses on this critical error sensitivity issue. Preschool children were given a classic version of a number conservation task in which an intuitively cued response conflicted with the correct conservation response and a control version in which this conflict was not present. After solving each version children were asked to indicate their response confidence. Results showed that in contrast with children who gave a correct conservation response, preschoolers who erred showed a sharp confidence decrease after solving the classic conflict problem. This suggests that nonconserving preschoolers detect that their response is questionable and are less ignorant about conservation than their well-documented errors might have previously suggested.
\end{abstract}

\section{Introduction}

A critical transition in children's cognitive development is the acquisition of number conservation. This key principle boils down to the insight that a numerical quantity will remain the same despite adjustment of its apparent shape or size. For example, imagine you are presented with a row of coins that is subsequently being stretched out. Adults and older children will have little trouble grasping that although the row will be longer when stretched out, the stretching does not alter the number of coins, of course. However, since the seminal work of Piaget [1] it is well established in the developmental literature that young children (i.e., until approximately age seven) typically fail this task and seem to be convinced that the longer row also contains more coins (e.g., see [2-4]).

Given the importance of number conservation for a child's numerical and mathematical development it is not surprising that children's conservation errors have been studied extensively. In the work of Piaget [1], who introduced the coin-spreading-task, number conservation marked a critical transition from a preoperational to operational stage in children's thinking. According to Piaget, the nonconserving preoperational child cannot grasp the conservation principle because they are limited to a purely intuitive and perceptual way of processing information. That is, in the coin spreading task the preoperational child will base its judgment purely on the visuospatial property of length. Consequently, they are bound to be misled by a length-equals-number intuition.

More contemporary developmental research has stressed the role of inhibitory processing [5-10]. According to these accounts young children's notorious conservation task failures do not necessarily reflect a categorical inability to grasp the number conservation principle per se but rather a failure to override their erroneous visuospatial intuition. Children's limited executive resources would not allow them to succeed in this demanding override process.

In general, inhibitory developmental accounts have received wide support and have become increasingly popular (e.g., $[3,6,11-18]$. However, the precise nature of children's inhibition failure in the number conservation task is not 
clear. A critical but perhaps somewhat overlooked question is whether children fail the task because they lack the executive resources to complete the inhibition process or because they fail to detect that they need to inhibit in the first place. To clarify this point it is important to stress that inhibitory accounts do not posit that children always need to block their visuospatial impressions (e.g., [8, 17, 19-24]). Often our visuospatial impression provides a valid and useful basis for our judgment. Indeed, in a lot of everyday situations length and quantity will typically covary. For example, when there are two rows of people waiting in line at the super market or two rows of cars standing before a red light, the longer row will typically also contain more items. In a number conservation task the visuospatial impression needs to be overridden because it results from a mere transformation and violates the logical conservation principle. This implies that an efficient inhibition requires that one monitors for such conflict first and inhibits the visuospatial impression whenever it is detected. The detection might be quite implicit and might boil down to a vague awareness that the visuospatial intuition is not fully warranted (e.g., [25-27]) but it is nevertheless a crucial building block for an efficient inhibition process. Hence, what we need to know is whether children show some minimal awareness of the questionability of their conservation errors or not. Unfortunately, the efficiency of such an error detection process in number conservation has not been directly examined.

From a theoretical point of view, testing children's conservation error detection skills is paramount to unravel the precise nature of their number conservation error. However, at a more applied level establishing whether or not children have some basic sensitivity with respect to their conservation errors is also important to develop efficient intervention programs to de-bias their judgment. Existing general educational intervention programs aimed at reducing children's and adults' overreliance on intuitive impressions during reasoning have often focused on training participants' inhibitory processing capacities (e.g., [14, 28-31]). However, if younger children do not yet detect that their intuitive length-equals-number response is erroneous, such inhibition training will have less than optimal results in the case of number conservation. Clearly, any increase in inhibitory processing capacity per se is rather pointless if one is not able to determine whether or not it is needed to inhibit in the first place. Hence, examining children's conservation error detection skills is paramount to determine which component an optimal intervention program needs to target.

In sum, both for theoretical and practical reasons it is important to test children's conservation error detection efficiency. In the present study we directly address this issue. We focused on the performance of preschoolers since children at this age level typically fail the number conservation task. To test our hypothesis, children were given both a classic version of the number conservation task in which the intuitively cued visuospatial length-equals-number response conflicted with the correct conservation response (i.e., conflict version) and a control or no-conflict version in which this conflict was not present. That is, in the conflict version children initially see two rows of equal length containing the same number of coins. Next, one of the rows is spread apart so that one is longer than the other and children are asked whether the two rows contain the same number of coins. In the no-conflict version the two rows also have the same number of coins but initially differ in length. The longer row is now transformed (i.e., contracted) to give both rows equal length and the child is asked whether the two rows contain an equal number of coins. Hence, the critical difference is that the control problem does not cue an erroneous visuospatial response.

After solving each version children were asked to indicate their response confidence on a simplified rating scale. This allowed us to measure children's error detection sensitivity. If preschoolers do not have an elementary understanding of the conservation principle or do not detect a conflict between their erroneous intuitive answer and this knowledge, their response confidence should not differ after solving conflict and no-conflict problems. However, if children have a minimal awareness of the unwarranted nature of their conservation error, this should decrease their confidence and result in lower confidence ratings after solving conflict than after solving no-conflict control problems.

\section{Method}

2.1. Participants. Forty-two preschoolers (mean age $=5.73$ years, $\mathrm{SD}=.29,25$ girls) were tested. The children were recruited from two childcare centers in Caen, France. They were all from middle-class homes and all had French as their mother tongue. The study was approved by the local school board and all parents or guardians gave informed consent for the study.

2.2. Material and Procedure. All participants were tested individually in a quiet room. Before starting the experiment, the children were familiarized with the confidence scale. A 5point rating scale (see Figure 1) was printed on an A4 sheet. On top of the scale children saw a cartoon character that was scratching his head and seemed to be in doubt. Five "smileys" were used to represent the different levels of the scale that ranged from 1 ("really not sure") to 5 ("totally sure"). The experimenter presented the scale and explained each point and asked the child to reexplain the scale. To facilitate the understanding of the scale, the experimenter also asked a set of trivia questions of variable difficulty (e.g., "What's your name? Does the cat meow or bark? $2+3=5$ ? Is Brussels a town in France?"). The children gave their responses by pointing on the smiley that best reflected their feeling of confidence.

The precise instructions that were used to introduce the rating scale were as follows:

Here you see some smileys and frownies. I'll explain you what they mean. They'll allow you to tell me whether you're sure or not sure about the answers you give me.

This one (experimenter points to first emoticon) means that you're really not sure that your answer is correct. You see this little mister 


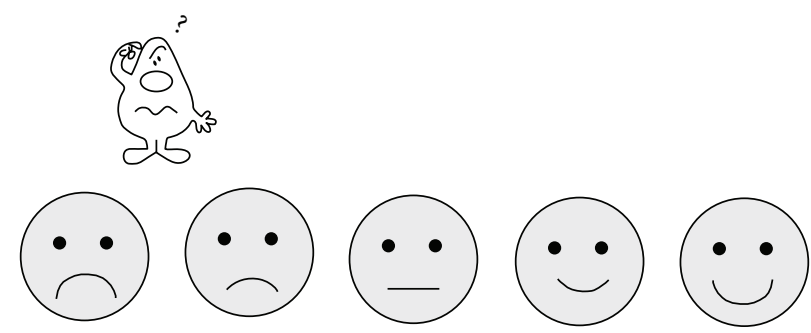

FIGURE 1: The five-point confidence scale. Five smileys were used to represent the different levels of the scale that ranged from 1 ("really not sure") to 5 ("totally sure").

with a big frowny face who really, really does not look to be sure about his answer. This one (experimenter points to second emoticon) means that you're not sure that your answer is correct. You see this little mister with a frowny face who does not look to be sure about his answer. This one (experimenter points to third emoticon) means that you're either sure, nor unsure that your answer is correct. You see this little mister who is neither sure nor unsure about his answer. This one (experimenter points to fourth emoticon) means that you're sure that your answer is correct. You see this little mister with a smiley face who looks to be sure about his answer. This one (experimenter points to fifth emoticon) means that you're really sure that your answer is correct. You see this little mister with a big smiley face who looks to be really, really sure about his answer. Hence, the more you go that way (experimenter points to the right), the more you are really sure about the answer that you are giving me. OK? Can you explain me what each face means, please? (Instructions are repeated until the child manages to explain each face). Now, I'm going to ask you some questions to see if you got everything.

(i) What's your name?

(ii) $2+3=5$ ?

(iii) Does the cat meow or bark?

(iv) Is Brussels a town in France?

(For each question, the child is presented with the scale and asked for a confidence judgment).

Note that the training questions were designed to be either very easy ("what is your name") or very hard ("Is 2 $+3=5$ ") for preschoolers. The idea was that by presenting questions of varying difficulty we could quite naturally illustrate the response-confidence concept by pointing out that one might be sure about their response to some questions, whereas one might be less sure about their response to other questions. For the hard questions, children were explicitly told by the experimenter that this was a really hard question and that they presumably felt unsure as to whether their answer was correct. We used this to demonstrate that in such a case they could express this on the rating scale by picking one of the "frownies" on the left-hand side. Overall, the familiarization indicated that children had little trouble understanding the confidence question and rating scale.

The children were presented with a computer version of the classical Piagetian conservation-of-number task (e.g., [32]). Stimuli were presented with Microsoft PowerPoint on a laptop computer with a 13 -inch screen. Each participant was positioned approximately $50 \mathrm{~cm}$ from the screen. Stimuli were displayed on a white computer screen which was split in two by a black horizontal line. There were two rows, one on each side of the line, always containing the same number of coins (i.e., 6 colored circles). All participants solved two versions of the task: a classic (i.e., conflict) version and a control (i.e., no-conflict) version (see Figure 2). As we explained, in the conflict version the two rows initially had the same length. Next, one of the rows was spread apart by apparent movement on the computer screen so that one was longer than the other and children were asked by the experimenter whether the two rows contained the same number of coins. In the no-conflict version the presentation was reversed. Children initially saw two rows that differed in length. Next, the longer row was contracted by apparent movement on the screen to give both rows equal length and the child was asked by the experimenter whether the two rows contained an equal number of coins. Presentation order of the conflict and no-conflict problem was counterbalanced. Half of the participants started with the conflict version, whereas the other half started solving the no-conflict version. Children responded verbally to the numerical equivalence question and were presented with the confidence scale and question immediately after they gave their answer.

After the first presentation of the problems, the same problems were shown a second time and a typical Piagetian countersuggestion was proposed. That is, the children were told that another child had given the opposite answer (e.g., "...but another little boy (girl) just told me that the number of coins was (not) the same...") and were asked whether they still believed that the number of coins was the same or not. Piaget used this countersuggestion in his classification of conserving and nonconserving participants. Only children who explicitly resisted the countersuggestion (i.e., who stuck to their original response) were classified as true conservers or nonconservers. We included the procedure to validate our findings against this classical Piagetian criterion. A total of 38 out of 42 participants indeed resisted the countersuggestion. None of our findings was affected when the four nonresisters 


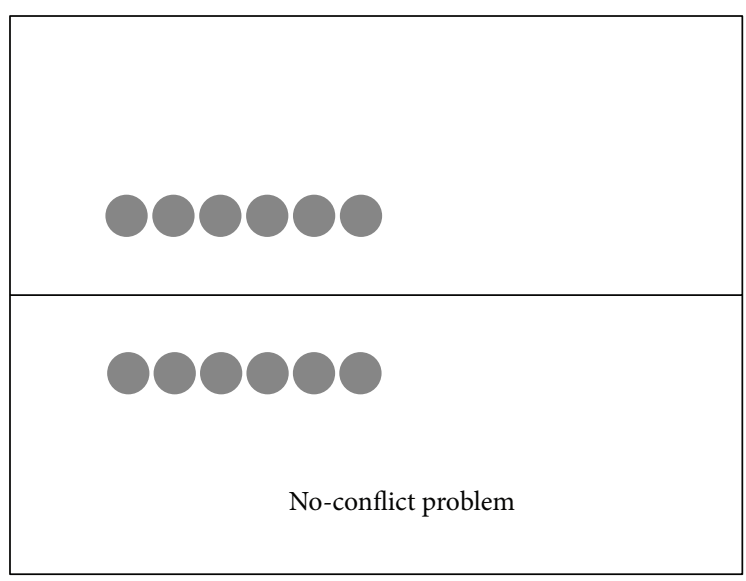

(a)

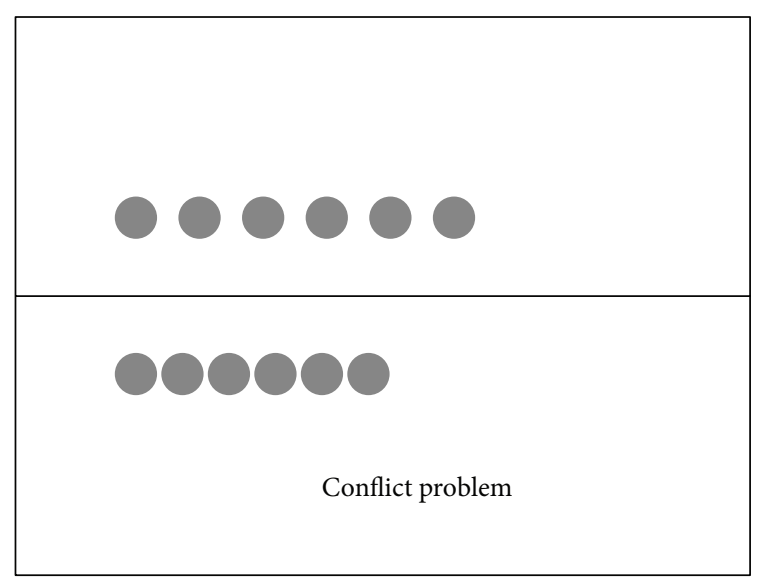

(b)

FIGURE 2: Overview of the material used for the no-conflict and conflict problem. Note that the figure illustrates the "end state" of the problems after the transformation.

were excluded from the analyses. All reported data in the result section concern the full sample of 42 participants.

\section{Results and Discussion}

3.1. Accuracy. Consistent with previous findings, about $60 \%$ ( $\mathrm{SE}=8 \%)$ of our preschoolers failed to solve the classic problem version in which the spreading of the coins cued a misleading visuospatial impression that conflicted with the correct conservation response. However, as expected, when this conflict was not present, children had little difficulty solving the task. Accuracy on the control version reached $100 \%$, Wilcoxon $Z(42)=4.37, P<.00001$. This establishes that children's failure to solve the conflict problem cannot be attributed to a general lack of motivation or concentration.

3.2. Response Confidence. The central question in our study concerned participant's response confidence. Obviously, to test the error detection sensitivity we were specifically interested in the ratings of children who failed to solve our classic conservation task version (i.e., the nonconservers, $n=25$, 16 girls, mean age $=5.74$ years, $S D=.29$ ). For completeness, we also analyzed the data of the group of children who gave the correct conservation response (i.e., conservers, $n=17,9$ girls, mean age $=5.71$ years, $\mathrm{SD}=.29$ ) and included response group as an additional factor in our design. Confidence ratings were rescored as percentage scores and subjected to a 2 (group, between subjects) $\times 2$ (conflict version, within subject) ANOVA.

We observed a main effect of group, $F(1,40)=14.19$, $P<0.001, \eta^{2} p=0.26$, a main effect of the conflict factor, $F(1,40)=13.93, P<0.001, \eta^{2} p=0.26$, and a significant two-way interaction between the group and the conflict factors, $F(1,40)=16.02, P<0.0005, \eta^{2} p=0.29$. As Figure 3 shows, for the group of conservers confidence ratings were high throughout. Indeed, post hoc Bonferroni tests indicated that the group of conservers were as confident in their answer on the conflict version as in their control version

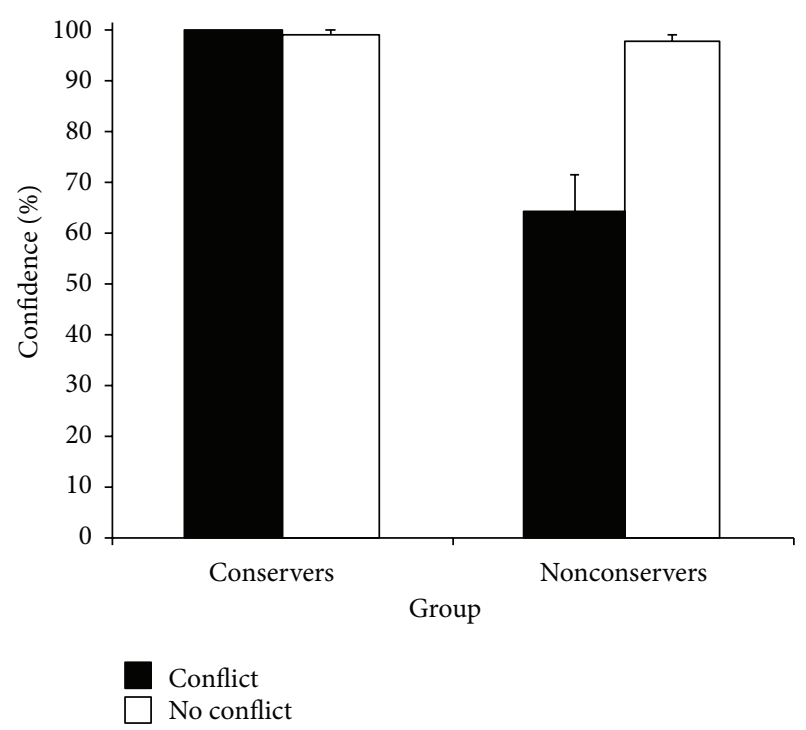

Figure 3: Average response confidence (\%) on conflict and noconflict problems in the group of conservers and nonconservers. Five-point confidence ratings were rescaled as percentage scores. Error bars are standard errors.

answer (99\% versus $100 \%$, ns, Cohen's $d=0.25$ ). Hence, preschoolers who manage to override the salient visuospatial intuitive response and answer the classic conservation task correctly also seem to know that their response is in fact correct. However, the critical data concerns the nonconservers. As Figure 3 shows, in contrast with the conservers, nonconservers were significantly less confident about their response on the conflict (64\%) problem than on the noconflict (98\%) problem, $P<.00001$, Cohen's $d=1.53$. Hence, although the nonconservers did not manage to give the correct response, their confidence indicates that they are not completely oblivious to their error. Recall that the key difference between the control and conflict versions was whether the visuospatial intuition conflicted with the correct 
conservation response or not. If nonconservers were not to have some elementary understanding of the conservation principle or were not detecting a conflict between their erroneous intuitive answer and this knowledge, they should have no reason to doubt their answer.

In this respect it is important to stress, as Figure 3 also indicates, that the lower confidence for conservers versus nonconservers was only observed on the conflict problems (64\% versus $100 \%, P<.00001$, Cohen's $d=1.36$ ) and not on the no-conflict problems (98\% versus $99 \%$, ns, Cohen's $d=.20$ ). Hence, it is not the case that nonconservers simply are overall less confident about their decisions than conservers. The lower confidence only occurs in the condition where giving the correct response requires dealing with a misleading visuospatial length-equals-number intuition. In sum, although nonconservers did not manage to inhibit this intuitive response our confidence data directly indicate that they at least detect that it is questionable.

A critic might note that our findings could be confounded by the within-subject presentation of the conflict and noconflict problems. That is, the contrast with the no-conflict problem might have specifically alerted children to the conflict manipulation and boosted their detection performance. Clearly, our newly designed control problem is not presented in a classic Piagetian number conservation task. Hence, it might still be the case that nonconservers show no error detection in the classic task. To test this confound hypothesis we entered presentation order (i.e., first conflict or first noconflict) as an additional between-subjects factor in our design. However, neither the main effect of presentation order nor its interactions with the other factors reached significance (all $F<1$ ). In addition, we also ran an analysis that was restricted to the first problem participants solved (i.e., remember that this was a conflict problem for half of the participants and a no-conflict problem for the other half). Hence, any within subject presentation confound is by definition excluded. Results were completely consistent with our main analysis. There was a main effect of group, $F(1,38)=11.32, P<0.0025, \eta^{2} p=0.23$, a main effect of the conflict factor, $F(1,38)=9.69, P<0.005, \eta^{2} p=0.20$, and a significant two-way interaction between the group and the conflict factors, $F(1,38)=9.96, P<0.005, \eta^{2} p=$ 0.20 . Bonferroni tests indicated that participants who failed to solve the conflict problem showed lower confidence than participants who solved the no-conflict control problem $(58 \%$ versus $99 \%, P<.0005$, Cohen's $d=1.5)$ and participants who solved the conflict problem correctly (58\% versus $100 \%$, $P<.0005$, Cohen's $d=1.56)$.

\section{General Discussion}

The present findings establish that nonconserving preschoolers are detecting their number conservation error. Although preschoolers typically fail to provide a correct conversation response and fall trap to the length-equals-number intuition, their decreased response confidence indicates that they at least detect that their response is not fully warranted. These data directly argue against Piaget's classic characterization of the nonconserving child as a preoperational, illogical reasoner who is bound to rely on mere visuospatial intuitions. If nonconservers would not have some elementary understanding of the conservation principle they should have no reason to doubt their answer. The findings further imply that in more recent accounts that stipulate that conservation errors result from children's inability to successfully inhibit their length-equals-number intuition, the inhibition failure should be conceived as a failure to complete the inhibition process rather than as a failure to detect that inhibition is required. Indeed, the present findings directly establish that nonconserving preschoolers have no trouble noticing that their erroneous conservation response is questionable per se.

Obviously, we do not claim here that nonconserving preschoolers have a fully explicit understanding of the conversation principle that they can verbally justify. We are referring to an elementary and implicit mastering that suffices to signal the questionability of the visuospatial intuitive response. That is, it is assumed that preschoolers sense that their response is erroneous but this does not imply that they will also manage to explicitly label this feeling and explain why their answer is wrong, for example. Note that such lack of error explicitation has also been observed in other fields (e.g., $[25,26,33,34])$. In the present context, the implicit nature of children's error detection is presumably also reflected in their responses to our Piagetian counter suggestion question. Recall that in our study we incorporated this classic procedure and told children after they gave their conservation task response that another child had just given the opposite answer (e.g., "...but another little boy (girl) just told me that the number of coins was (not) the same..."). Children were then asked whether they wanted to change their answer. As in Piaget's studies, we observed that the vast majority of children resisted the countersuggestion and stuck to their original response. That is, the same children who were doubting their erroneous conservation response, as evidenced by their confidence ratings, could not be persuaded to explicitly alter it. Although Piaget's countersuggestion might suffer from sociopsychological confounds (i.e., it requires that one is able to overtly admit that one was wrong) it at least suggests that children's doubt is not easily reflected in more explicit behavioral measures.

As a side note, we believe that the typical countersuggestion findings are one factor that might have blurred Piaget's view and contributed to his strong categorical claims about the mere intuitive processing and lack of number conservation knowledge in preoperational children. The fact that nonconservers typically resist the countersuggestion and do not alter their response might have given the false impression that they show no doubt and are highly confident that their intuitive response is correct. The use of a more direct and subtle confidence measure in the present study allowed us to rectify this view. This clarifies that an exclusive reliance on countersuggestion as an index of response confidence is bound to result in an underestimation of preschoolers' cognitive and logical skills.

It might be interesting to link the present work on number conservation to research on bias detection during logical and probabilistic thinking in the reasoning and decision-making 
field. Classic studies on reasoning and decision-making have long established that people's inferences are often biased by prior beliefs and stereotypical intuitions (e.g., $[35,36])$. In line with the present findings, it has recently been shown that reasoners also detect the biased nature of their intuitive logical and probabilistic judgments (e.g., [21, 25, 37-42]). Interestingly, however, developmental studies have suggested that this bias detection during logical and probabilistic reasoning is only observed after the onset of adolescence (i.e., by the end of elementary school, e.g., $[43,44])$. This developmental pattern has been linked to the late maturation of the Anterior Cingulate Cortex (ACC), the critical brain structure that is supposed to be mediating conflict and error detection, which only achieves full functionality over the adolescent years (e.g., [45-48]). Given these findings, the presently established successful number conservation error detection at the preschool age might seem somewhat surprising. However, here we need to take into account that a less developed ACC does not imply a lack of all conflict detection. Indeed, basic error monitoring studies have shown that even three-year olds can detect errors in simple tasks that do not cue a strong intuitive response [49]. Arguably, in comparison with logical and probabilistic reasoning tasks in which the cued intuitive response typically entails a semantic prior belief or stereotypical information, the critical physical transformation in conservation tasks might act as a cue that directs children's attention and thereby facilitates monitoring. Hence, detection of intuitive bias in number conservation might be less demanding and occur at a younger age than in logical and probabilistic reasoning tasks. Although this prediction remains to be tested directly, it does suggest that a promising agenda for future neuroscientific studies on error monitoring and Anterior Cingulate Cortex development might be to more specifically contrast children's performance across different domains.

We noted that the present study has also implications with respect to the design of intervention programs. For example, the evidence for preschoolers' conservation error sensitivity indicates that there is little point in running programs that focus on a familiarization and teaching of the conservation principle per se. Bluntly put, if the problem is not that children do not know the conservation principle, merely informing them about conservation will not be very helpful. Rather, a more promising approach seems to be to focus on training children's capacities to override their erroneous visuospatial intuitions. As we noted, existing inhibitory training programs have been shown to be successful at reducing older children's and adults' overreliance on intuitive impressions during reasoning and decision making (e.g., [14, 28-31]). Since the present evidence suggests that preschoolers can reliably distinguish between situations in which their visual impressions violate conservation or not, such inhibitory training programs might prove to be highly efficient to boost preschoolers' logicomathematical performance.

\section{Conflict of Interests}

The authors declare that there is no conflict of interests regarding the publication of this paper.

\section{Acknowledgment}

Preparation of this paper was supported by a grant from the French National Research Agency (ANR-12-JSH2-0007-01).

\section{References}

[1] J. Piaget, The Child's Conception of Number, Routledge \& Kegan Paul, New York, NY, USA, 1952.

[2] G. Borst, N. Poirel, A. Pineau, M. Cassotti, and O. Houdé, "Inhibitory control in number-conservation and class-inclusion tasks: a neo-Piagetian inter-task priming study," Cognitive Development, vol. 27, pp. 283-298, 2012.

[3] O. Houdé, "Rationality in reasoning: the problem of deductive competence and the inhibitory control of cognition," Current Psychology of Cognition, vol. 16, pp. 108-113, 1997.

[4] R. M. Ping and S. Goldin-Meadow, "Hands in the air: using iconic gestures to teach children conservation of quantity," Developmental Psychology, vol. 44, no. 5, pp. 1277-1287, 2008.

[5] D. F. Bjorklund and K. K. Harnishfeger, "The resources construct in cognitive development: diverse sources of evidence and a theory of inefficient inhibition," Developmental Review, vol. 10, no. 1, pp. 48-71, 1990.

[6] F. N. Dempster and C. J. Brainerd, Interference and Inhibition in Cognition, Academic Press, San Diego, Calif, USA, 1995.

[7] O. Houdé, "Inhibition and cognitive development: object, number, categorization, and reasoning," Cognitive Development, vol. 15, no. 1, pp. 63-73, 2000.

[8] O. Houdé and E. Guichart, "Negative priming effect after inhibition of number/length interference in a Piaget-like task," Developmental Science, vol. 4, no. 1, pp. 119-123, 2001.

[9] G. Leroux, M. Joliot, S. Dubal, B. Mazoyer, N. Tzourio-Mazoyer, and O. Houdé, "Cognitive inhibition of number/length interference in a Piaget-like task in young adults: evidence from ERPs and fMRI," Human Brain Mapping, vol. 27, no. 6, pp. 498-509, 2006.

[10] G. Leroux, J. Spiess, L. Zago et al., "Adult brains don't fully overcome biases that lead to incorrect performance during cognitive development: an fMRI study in young adults completing a Piaget-like task," Developmental Science, vol. 12, no. 2, pp. 326338, 2009.

[11] R. Babai, R. R. Eidelman, and R. Stavy, "Preactivation of inhibitory control mechanisms hinders intuitive reasoning," International Journal of Science and Mathematics Education, vol. 10, no. 4, pp. 763-775, 2012.

[12] W. De Neys and D. Everaerts, "Developmental trends in everyday conditional reasoning: the retrieval and inhibition interplay," Journal of Experimental Child Psychology, vol. 100, no. 4, pp. 252-263, 2008.

[13] W. De Neys and E. Van Gelder, "Logic and belief across the lifespan: the rise and fall of belief inhibition during syllogistic reasoning," Developmental Science, vol. 12, no. 1, pp. 123-130, 2009.

[14] O. Houdé, "First insights on 'neuropedagogy of reasoning," Thinking \& Reasoning, vol. 13, no. 2, pp. 81-89, 2007.

[15] S. Moutier, S. Plagne-Cayeux, A.-M. Melot, and O. Houdé, "Syllogistic reasoning and belief-bias inhibition in school children: evidence from a negative priming paradigm," Developmental Science, vol. 9, no. 2, pp. 166-172, 2006.

[16] N. Poirel, G. Borst, G. Simon et al., "Number conservation is related to children's prefrontal inhibitory control: an fMRI study of a Piagetian task," PLoS ONE, vol. 7, Article ID e40802, 2012. 
[17] V. F. Reyna, F. J. Lloyd, and C. J. Brainerd, "Memory, development, and rationality: an integrative theory of judgement and decision-making," in Emerging Perspectives on Judgment and Decision Research, S. Schneider and J. Shanteau, Eds., pp. 201245, Cambridge University Press, New York, NY, USA, 2003.

[18] M. Simoneau and H. Markovits, "Reasoning with premises that are not empirically true: evidence for the role of inhibition and retrieval," Developmental Psychology, vol. 39, no. 6, pp. 964-975, 2003.

[19] C. J. Brainerd and V. F. Reyna, "Fuzzy-trace theory: dual processes in memory, reasoning, and cognitive neuroscience," in Advances in Child Development and Behavior, H. W. Reese and R. Kail, Eds., vol. 28, pp. 41-100, Academic Press, San Diego, Calif, USA, 2001.

[20] J. E. Jacobs and P. A. Klaczynski, "The development of judgment and decision making during childhood and adolescence," Current Directions in Psychological Science, vol. 11, no. 4, pp. 145-149, 2002.

[21] W. De Neys and S. Franssens, "Belief inhibition during thinking: not always winning but at least taking part," Cognition, vol. 113, no. 1, pp. 45-61, 2009.

[22] W. De Neys and K. Vanderputte, "When less is not always more: stereotype knowledge and reasoning development," Developmental Psychology, vol. 47, no. 2, pp. 432-441, 2011.

[23] P. A. Klaczynski, J. P. Byrnes, and J. E. Jacobs, "Introduction to the special issue: the development of decision making," Journal of Applied Developmental Psychology, vol. 22, no. 3, pp. 225-236, 2001.

[24] K. E. Stanovich, R. F. West, and M. E. Toplak, "The complexity of developmental predictions from dual process models," Developmental Review, vol. 31, no. 2-3, pp. 103-118, 2011.

[25] W. de Neys, "Bias and conflict: a case for logical intuitions," Perspectives on Psychological Science, vol. 7, no. 1, pp. 28-38, 2012.

[26] W. De Neys, "Conflict detection, dual processes, and logical intuitions: some clarifications," Thinking \& Reasoning, 2013.

[27] T. Proulx, M. Inzlicht, and E. Harmon-Jones, "Understanding all inconsistency compensation as a palliative response to violated expectations," Trends in Cognitive Sciences, vol. 16, no. 5, pp. 285-291, 2012.

[28] O. Houde, L. Zago, E. Mellet et al., "Shifting from the perceptual brain to the logical brain: the neural impact of cognitive inhibition training," Journal of Cognitive Neuroscience, vol. 12, no. 5, pp. 721-728, 2000.

[29] S. Moutier, "Deductive reasoning and experimental matchingbias inhibition training in school children," Cahiers de Psychologie Cognitive, vol. 19, no. 4, pp. 429-452, 2000.

[30] S. Moutier, N. Angeard, and O. Houdé, "Deductive reasoning and matching-bias inhibition training: evidence from a debiasing paradigm," Thinking \& Reasoning, vol. 8, pp. 205-224, 2002.

[31] S. Moutier and O. Houdé, "Judgement under uncertainty and conjunction fallacy inhibition training," Thinking \& Reasoning, vol. 9, no. 3, pp. 185-201, 2003.

[32] O. Houdé, A. Pineau, G. Leroux et al., "Functional magnetic resonance imaging study of Piaget's conservation-of-number task in preschool and school-age children: a neo-Piagetian approach," Journal of Experimental Child Psychology, vol. 110, no. 3, pp. 332-346, 2011.

[33] W. De Neys and T. Glumicic, "Conflict monitoring in dual process theories of thinking," Cognition, vol. 106, no. 3, pp. 12481299, 2008.
[34] J. St. B. T. Evans and D. E. Over, Rationality and Reasoning, Psychology Press, Hove, UK, 1996.

[35] J. S. B. T. Evans, "Intuition and reasoning: a dual-process perspective," Psychological Inquiry, vol. 21, no. 4, pp. 313-326, 2010.

[36] D. Kahneman and A. Tversky, "On the psychology of prediction," Psychological Review, vol. 80, no. 4, pp. 237-251, 1973.

[37] C. Bonner and B. R. Newell, "In conflict with ourselves? An investigation of heuristic and analytic processes in decision making," Memory \& Cognition, vol. 38, no. 2, pp. 186-196, 2010.

[38] W. De Neys and J. F. Bonnefon, "The whys and whens of individual differences in thinking biases," Trends in Cognitive Sciences, vol. 17, pp. 172-178, 2013.

[39] W. De Neys, E. Moyens, and D. Vansteenwegen, "Feeling we're biased: autonomic arousal and reasoning conflict," Cognitive, Affective \& Behavioral Neuroscience, vol. 10, no. 2, pp. 208-216, 2010.

[40] W. De Neys, S. Rossi, and O. Houdé, "Bats, balls, and substitution sensitivity: cognitive misers are no happy fools," Psychonomic Bulletin \& Review, vol. 20, pp. 269-273, 2013.

[41] K. Morsanyi and S. J. Handley, "Logic feels so good-I like it! Evidence for intuitive detection of logicality in syllogistic reasoning," Journal of Experimental Psychology, vol. 38, no. 3, pp. 596-616, 2012.

[42] E. J. N. Stupple and L. J. Ball, "Belief-logic conflict resolution in syllogistic reasoning: inspection-time evidence for a parallelprocess model," Thinking \& Reasoning, vol. 14, no. 2, pp. 168-181, 2008.

[43] W. de Neys, S. Cromheeke, and M. Osman, "Biased but in doubt: conflict and decision confidence," PLOS ONE, vol. 6, no. 1, Article ID e15954, 2011.

[44] W. De Neys and V. Feremans, "Development of heuristic bias detection in elementary school," Developmental Psychology, vol. 49, pp. 258-269, 2013.

[45] P. L. Davies, S. J. Segalowitz, and W. J. Gavin, "Development of response-monitoring ERPs in 7- to 25-year-olds," Developmental Neuropsychology, vol. 25, no. 3, pp. 355-376, 2004.

[46] W. De Neys, O. Vartanian, and V. Goel, "Smarter than we think: when our brains detect that we are biased," Psychological Science, vol. 19, no. 5, pp. 483-489, 2008.

[47] K. D. Fitzgerald, S. C. Perkins, M. Angstadt et al., "The development of performance-monitoring function in the posterior medial frontal cortex," NeuroImage, vol. 49, no. 4, pp. $3463-$ 3473, 2010.

[48] D. L. Santesso and S. J. Segalowitz, "Developmental differences in error-related ERPs in middle- to late-adolescent males," Developmental Psychology, vol. 44, no. 1, pp. 205-217, 2008.

[49] K. E. Lyons and S. Ghetti, "The development of uncertainty monitoring in early childhood," Child Development, vol. 82, no. 6, pp. 1778-1787, 2011. 

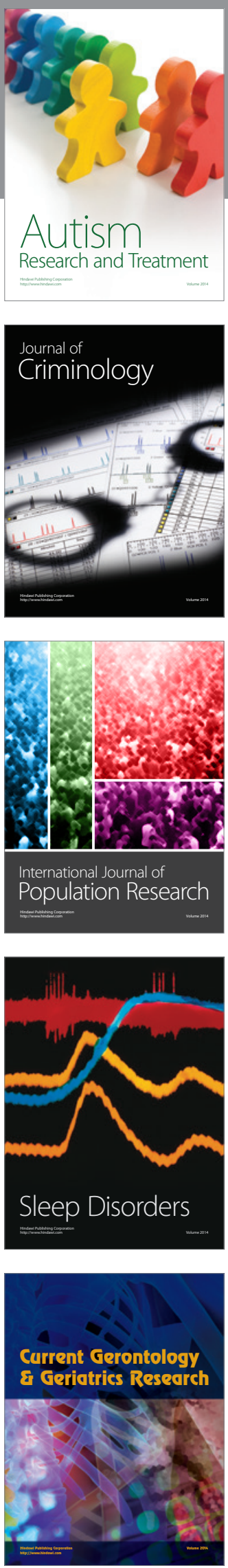
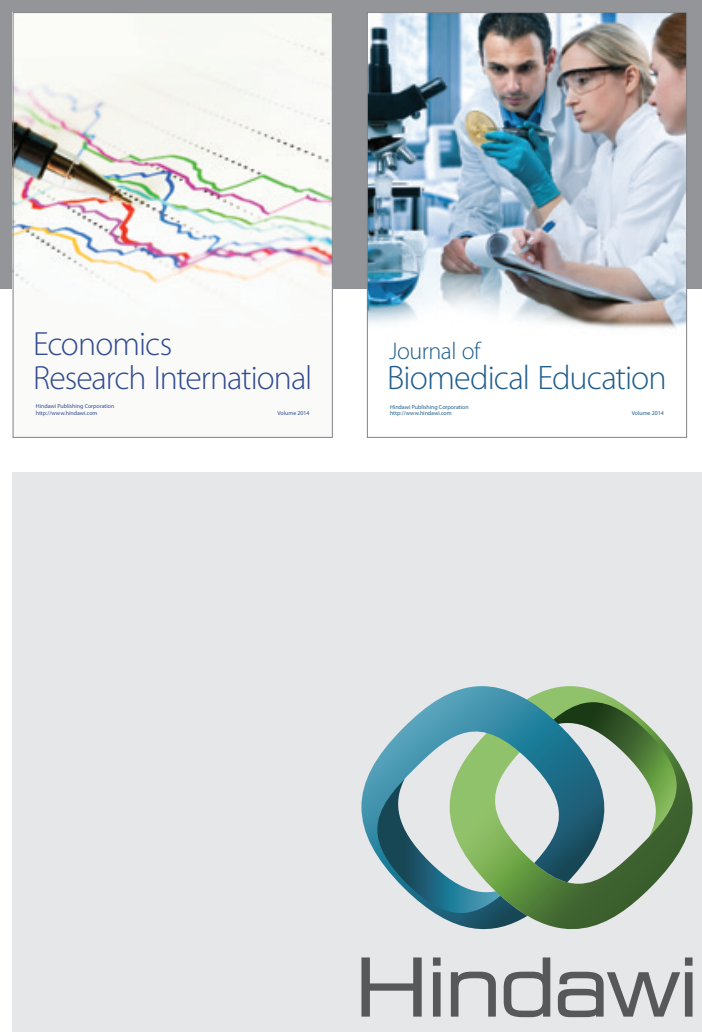

Submit your manuscripts at

http://www.hindawi.com
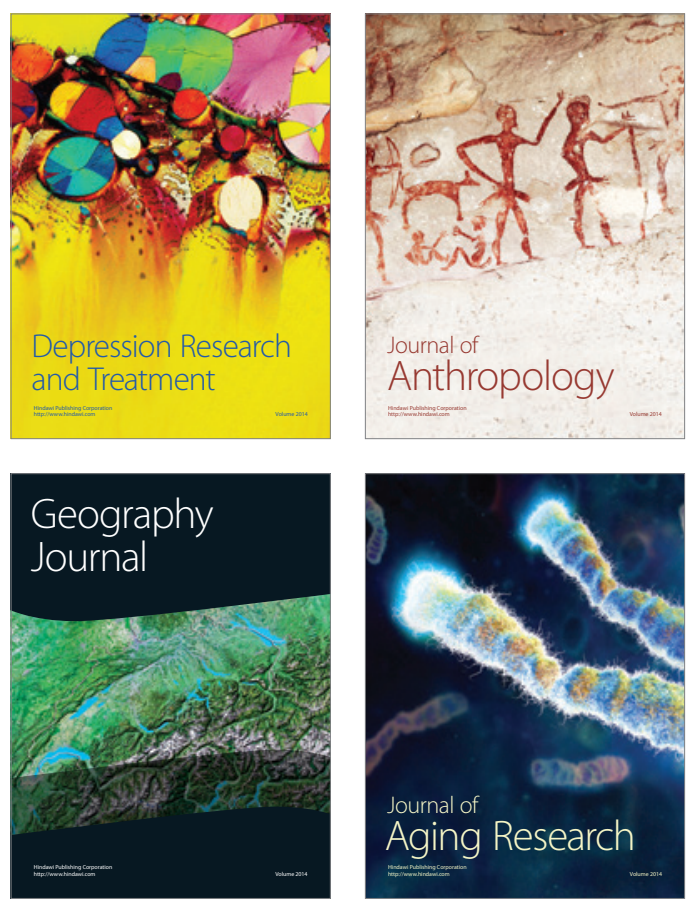
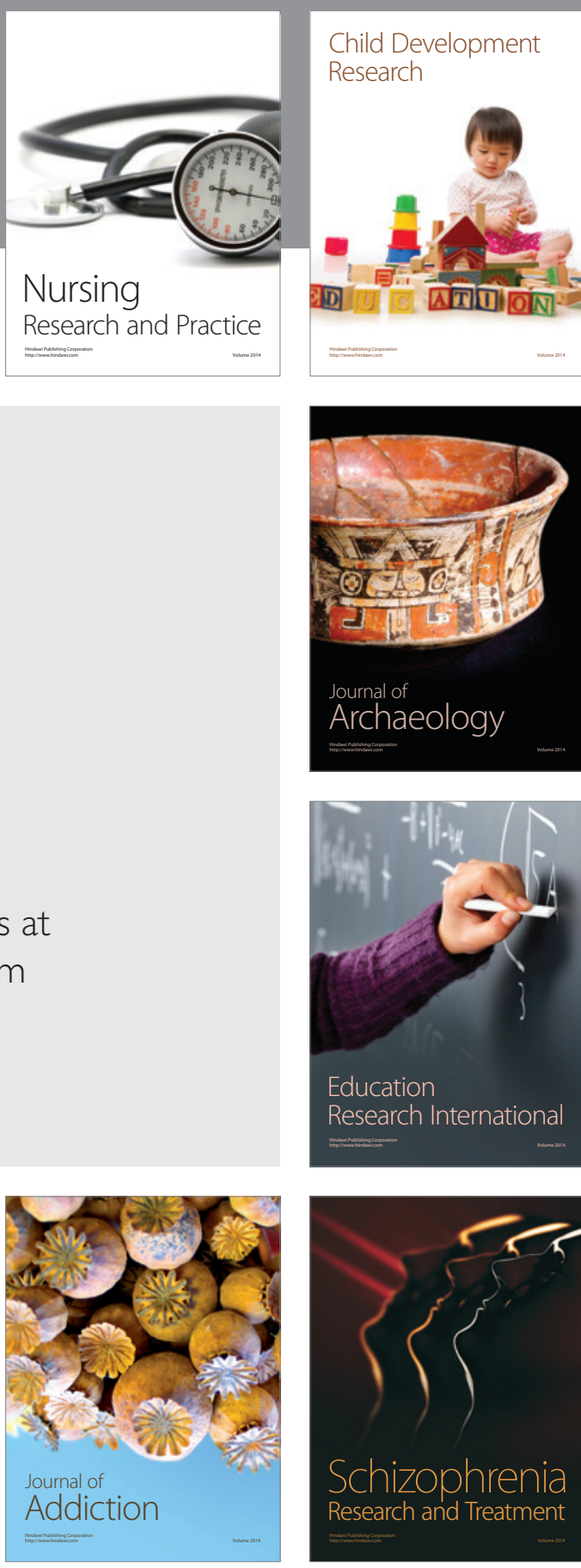

(D)
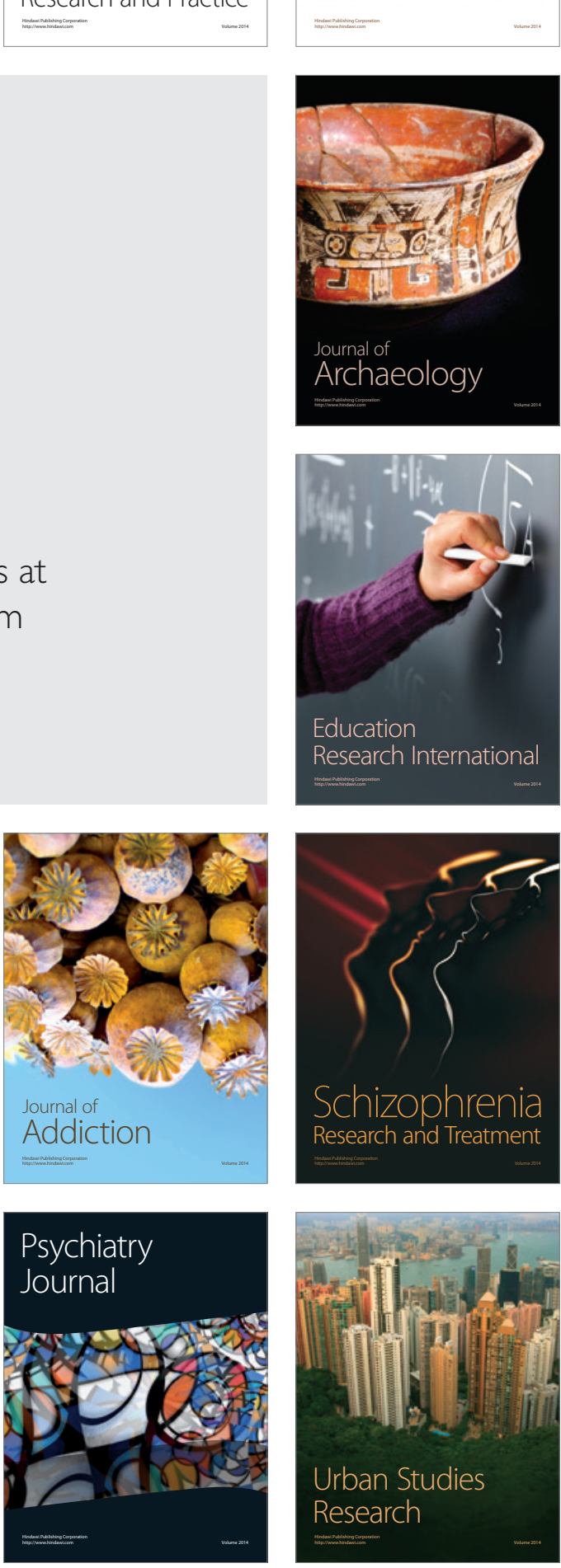